\title{
Intelligent warehouse monitoring based on distributed system and edge computing
}

\author{
Sen $\mathrm{Lin}^{1} \cdot$ Jianxin Huang ${ }^{2} \cdot$ Wenzhou Chen ${ }^{2} \cdot$ Wenlong Zhou ${ }^{3} \cdot$ Jinhong $\mathrm{Xu}^{2} \cdot$ Yong Liu $^{2} \cdot$ Jinqiang Yao $^{1}$
}

Received: 17 December 2020 / Accepted: 17 April 2021 / Published online: 1 May 2021

(c) The Author(s) 2021

\begin{abstract}
This paper mainly focuses on the volume calculation of materials in the warehouse where sand and gravel materials are stored and monitored whether materials are lacking in real-time. Specifically, we proposed the sandpile model and the point cloud projection obtained from the LiDAR sensors to calculate the material volume. We use distributed edge computing modules to build a centralized system and transmit data remotely through a high-power wireless network, which solves sensor placement and data transmission in a complex warehouse environment. Our centralized system can also reduce worker participation in a harsh factorial environment. Furthermore, the point cloud data of the warehouse is colored to visualize the actual factorial environment. Our centralized system has been deployed in the real factorial environment and got a good performance.
\end{abstract}

Keywords Edge computing $\cdot$ Point cloud projection $\cdot$ Volume estimation

\section{Introduction}

With the development of the internet, smart factories and smart warehouses (Liu et al. 2018; Lee et al. 2019; Scheer 2012) have gradually emerged. Machines have gradually replaced some simple and repetitive tasks. What's more, in some places with harsh production environments, unmanned people rapidly realize to reduce the human in such harsh environments.

In the harsh factory environment, most factories did not reserve some installing sensors, nor did they reserve the

S. Lin and J. Huang contributed equally to this work.

Jinqiang Yao

Sensorchina@aliyun.com

Sen Lin

Linsen220@126.com

Jianxin Huang

22032097@zju.edu.cn

1 Zhejiang Communications Group Inspection Technology Co., Ltd., Hangzhou, China

2 Department of Control Science and Engineering, Institute of Cyber Systems and Control, Zhejiang University, Hangzhou 310027, China

3 Hangzhou BigDataCloudAI Technology Co., Ltd., Hangzhou, China network cables needed for data transmission. The factory structure is pre-designed, and it is not easy to change the structure. The data transmission line will increase the cost very much and bring danger to the working environment, so we should find a way to monitor the warehouse without cable. The material volume calculation (Riccabona et al. 1995; Fojtík 2014; Zhongyi et al. 2019) of intelligent warehouse with distributed computing equipment proposed in this paper is used in the warehouse's harsh environment.

Two important concepts are involved: distributed systems and edge computing. Firstly, distributed systems have a wide range of applications in smart factories. A general feature of manufacturing systems is the transmission and processing of data between each other. Distributed systems provide coordination to allow global information to be available for further calculation and better decision making (Poonpakdee and Koiwanit 2018). The architectures of distributed systems we used are centralized systems, Fig. 1 presents the simulated scenarios. A server aims to centralize all functions and information taken from clients by directly connecting to the clients. Clients share their resources by sending and receiving the information to a server (Minar 2002). Secondly, edge computing is a distributed computing paradigm that brings computation and data storage closer to the location where it is needed to improve response times and save costs (Hamilton 2019). Edge computing is performed at the network edge near to the device or data source. Edge computing can 


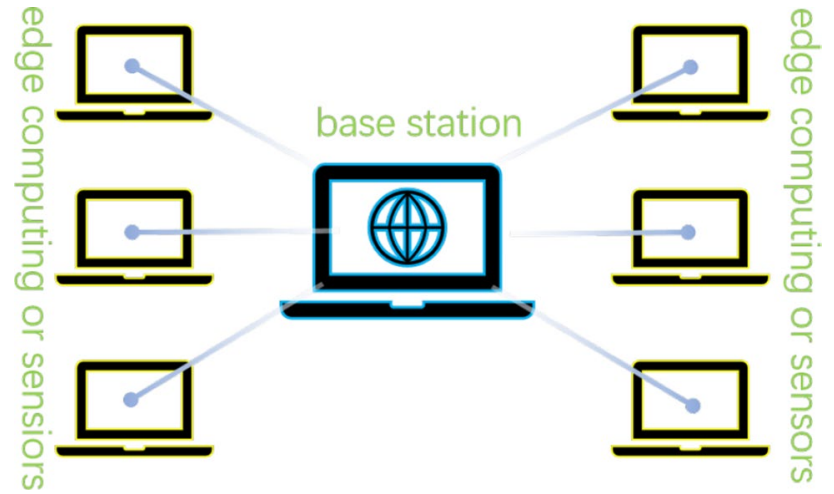

Fig. 1 Centralized systems. The systems have a base station and all sensors connect to it,base station can read data from every sensor,but sensors are independent

provide real-time data processing, data cleaning, and privacy protection (Shi et al. 2016). Pizoń and Lipski (2016) argued that edge computing enables dynamic monitoring and data process. Distributed computing is based on the rapid development of the network, and edge computing is related to factories' distributed automation. Edge computing combines decentralized processing with the centralized upload, which reduces data transmission time, data transmission errors, and data security while reducing bandwidth (Chen et al. 2017). The primary advantage of centralized systems is their simplicity. Shu et al. (2016) provides a method for collecting data and processing data by cloud-integrated. The Cyberphysical systems (CPSs) have three fundamental conflicting attributes: safety, security, and sustainability (Baheti and Gill 2011). For edge computing, De Brito et al. (2016) supposed that deployment of programmable fog nodes is attributed to inter-node Peer-to-Peer (P2P) communication and services orchestration without centralized control. The manufacturing industry is exploring the use of cloud computing to enhance manufacturing plant operations' efficiency, improve product quality, and so on (Georgakopoulos et al. 2016). However, the internet can be used in our environment, so the structure of distributed edge computing provided by others can not be used directly. We use a computer as a center and set up all edge computing modules to start automatically, and all of them are independent.

The distributed systems and edge computing are used in my system. There are severe dust and large-scale machinery operations in this kind of warehouse, but someone needs to monitor whether the materials in each warehouse are lacking. If the lack of materials needs to be supplemented in time, otherwise it will affect production efficiency. The edge computing equipment used in this article connects each computing module to a network through a wireless router (Ikram and Thornhill 2010; Paavola and Leiviska 2010) and monitors the volume of materials in the warehouse in real-time.
This article mainly contributes to the processing of distributed edge computing module data and the networking of distributed equipment. The module is mainly composed of a mini processor, Livox Horizon LiDAR, camera, and wireless router. After power on, each module will be connected to the network through the router, and the equipment can be started by remote login, and the central control room can also be obtained through high-power wireless access. The main contributions of this paper can be presented as follows:

1. Distributed edge computing devices transmit data through wireless network networking.

2. Adding materials through real-time volume calculation and intelligent notification to reduce personnel working in harsh environments.

3. The sand pile model is used to predict the sand piles that cannot be scanned by LiDAR, reducing the redundancy caused by installing sensors at the bottom of the warehouse.

\section{Method}

The intelligent material warehouse has two essential components: the edge computing end and the monitoring room. The edge computing is responsible for two parts, volume calculation and the third is point cloud colouring Fig. 15. Point cloud colouring makes the 3D point cloud with the information of the image can be displayed on the monitoring room. There is much dust in the material area in the actual warehouse. The measurement accuracy will be greatly affected, such as the ZED stereo camera, which is a depth camera based on the principle of RGB binocular stereo vision (Bauer et al. 2019), can meet $20 \mathrm{~m}$ distance. But it is difficult to meet the requirements of distance and accuracy, so we choose LiDAR to obtain three-dimensional data. Traditional line LiDAR can not get dense point cloud data, and the actual scene viewing angle is only 90 , so using 360 lasers make a waste of a performance. The Livox Horizon LiDAR is a non-repetitive LiDAR with a field of view (FOV) $81.7^{\circ}$ (horizontal) $\times 25.1^{\circ}$ (vertical). Two lasers are arranged on the left and right sides of the warehouse to achieve fullrange coverage. By accumulating multiple frames of LiDAR data, a denser point cloud data can be achieved. In order to splice the left and right point clouds, the transfer matrix from the left point cloud to the right point cloud needs to be calibrated. The left point cloud should be changed to the right point cloud frame by multiplying the transform matrix. After adding left and right point cloud, changing the adding point cloud to the map and then remove the plane and wall point by plane constrain. We want to transmit data to the monitoring room through wireless long distance for people, but it takes more bandwidth to transmit pictures. Hence, 
we color the point cloud and transmit the point cloud to the monitoring room, saving wireless bandwidth. The point cloud colouring technology (PDAL Contributors 2018) is equivalent to using LiDAR data and two-dimensional image data to make a data fusion to generate an image similar to the RGB-D image. The monitoring room can see the RGB-D similar data information. Another one, the coloured point cloud can be used for $3 \mathrm{D}$ reconstruction (Newcombe et al. 2011), in some places where RGB-D cameras are not convenient to use. The intelligent warehouse system can be broken down into several parts: hardware framework, volume calculation, LiDAR camera calibration and point cloud colouring, which will be introduced one by one.

\subsection{The hardware system}

The hardware system of the intelligent material warehouse includes a distributed edge computing module composed of the Jetson-nano development board, Livox Horizon LiDAR, Huawei WIFI3 AX3 wireless as Fig. 4a, b. Jetson-nano is a small processor, which comes with a small camera can be used to collect images, is responsible for LiDAR point cloud processing and image recognition. Huawei routers adopt wireless networking configuration information. The base station uses the auxiliary transceiver that can interact with the base station transceiver of the neighbouring cell base station to exchange network parameters with the neighbouring cell base station and configures their neighbouring cell lists based on this. Stations exchange and update of configuration information, and self-adaptive networking. After power on, the processor and the program will automatically start, and automatically connect to the wireless network. The module hardware system connection diagram is shown in Fig. 2.

The installation of edge computing modules in the warehouse is distributed as Fig. 3. The traditional sensor arrangement requires the LiDAR to be connected to the processor through a network cable. However, in a warehouse environment with a long distance, we need multiple LiDARs, so it is very inconvenient to connect the LiDAR to a network by pulling the network cable. The edge computing module are suitable for installation in this environment. Each edge computing module performs independent calculations, and the processor performance requirements for edge computing do not need to be very high. Combine all modules in a local area network through a router, and send data through a high-power wireless (Farkas 2011) transmitting device Fig. 4, The entire system is based on the Robot Operating System (ROS). ROS is a distributed framework which nodes can be combined through a loose coupling. The nodes can run on different computing platforms and communicate through topics. In the same ROS system, only a master is allowed, so set the host of the control room as the master node, and combine all other modules under the same local area network as the client. The volume of storage material warehouse is $20 \times 10 \times 4 \mathrm{~m}^{3}$, which is not easy to install edge computing modules, so choose to install the modules on the left and right columns in front of the warehouse. We will use the algorithm to predict the height of some materials that cannot be scanned to improve the accuracy of volume calculation.
Fig. 2 Module hardware connection diagram
Perceive Module structure

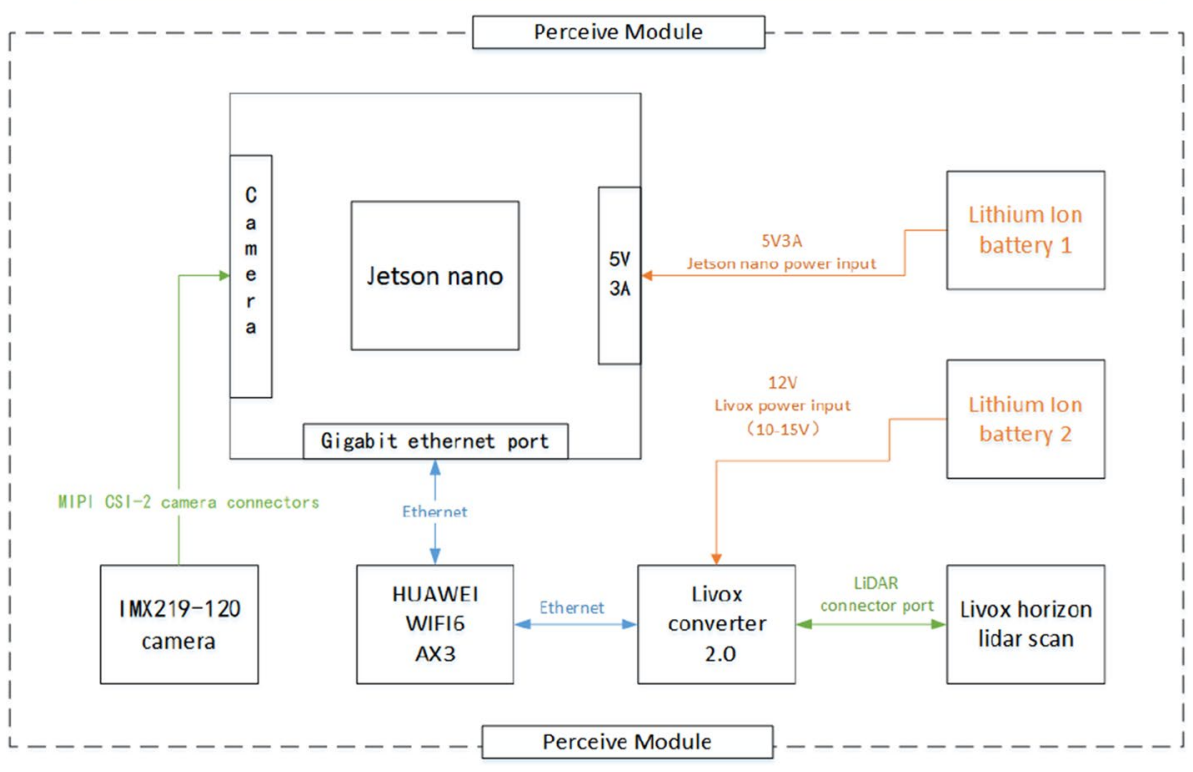


Fig. 3 The module installed on the warehouse

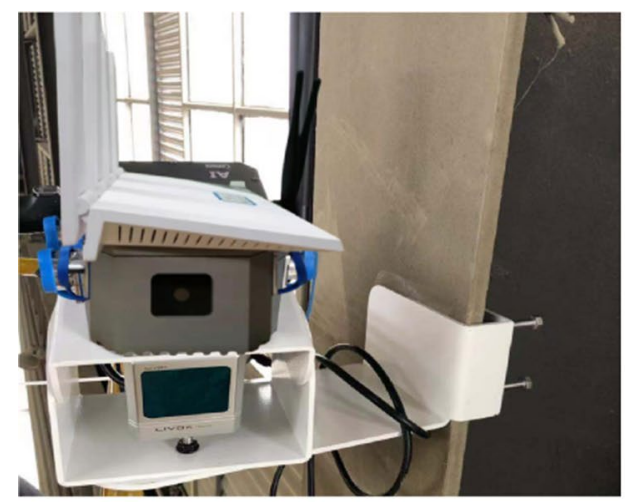

(a)

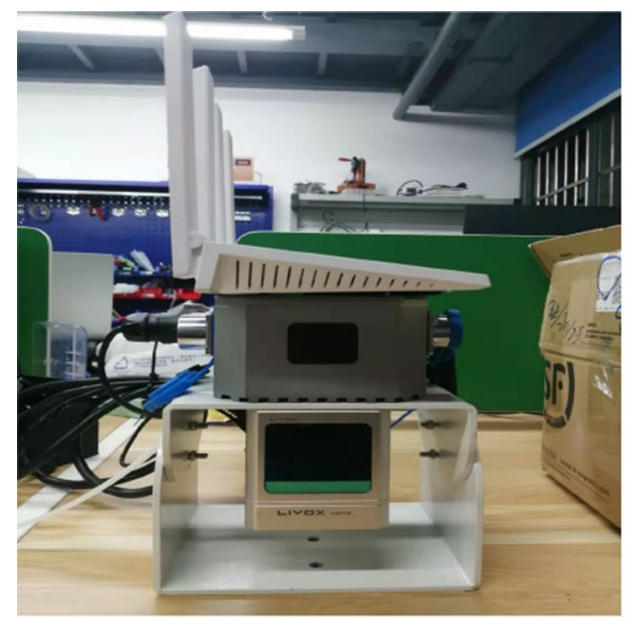

(a)

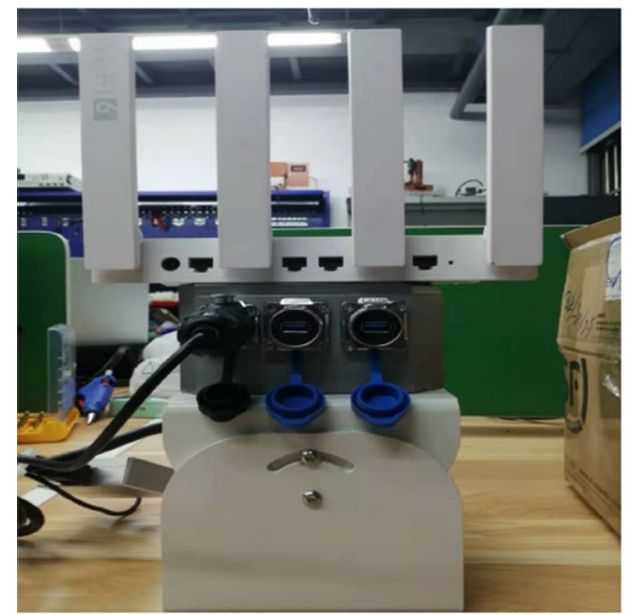

(c)

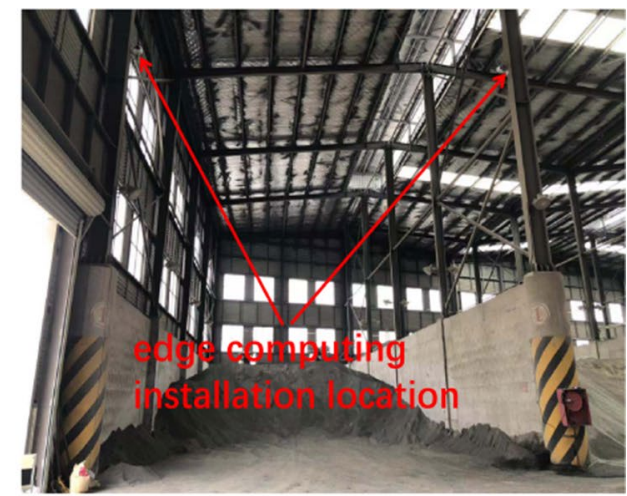

(b)

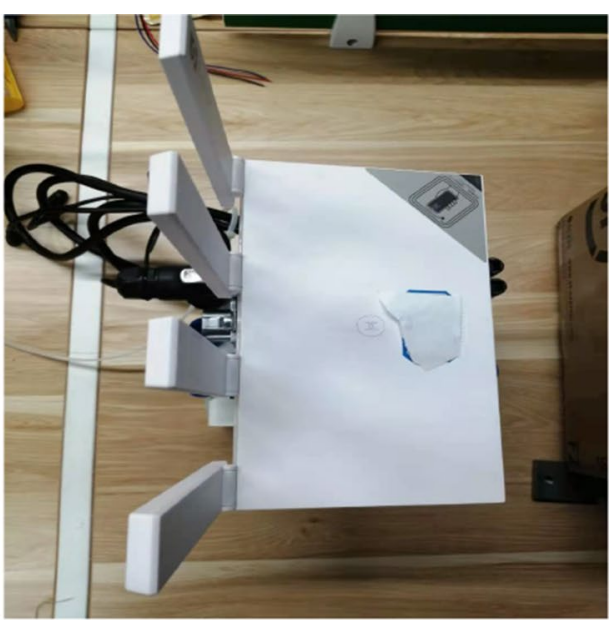

(b)

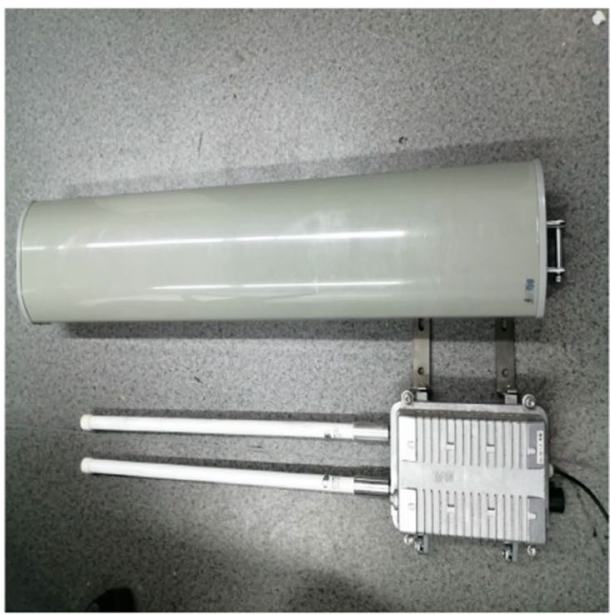

(d)

\subsection{The point cloud correction}

The point cloud correction has two parts, one is the point cloud correction of the left and right modules of the warehouse and splicing them into a complete point cloud. The other is the correction of the spliced point cloud to a prebuilt 3D map by LiDAR Odometry and Mapping (LOAM) (Zhang and Singh 2014). The LiDAR data of the modules 
on the left and right sides are a set of intersecting data and cannot be directly spliced into a complete warehouse point cloud data. It needs to be calibrated to find the transform of the left and right point clouds, and then the two sets of data are stitched together. We choose the right side as the source cloud and the left side as the target cloud, find the transform from left to right, rotate the point cloud on the left to match the right. The 3D point cloud map of the warehouse is constructed in advance, and the data collected in real-time is at the origin of the coordinates by default. Transform needs to be calibrated to transform the collected $3 \mathrm{D}$ point cloud to the corresponding warehouse of the collected data. Because the warehouse has a certain similarity and the established threedimensional model and the data have a large transformation distance, it cannot be automatically calibrated by the algorithm at one time, so there are two steps in the calibration. One is to manually adjust the point cloud data, adjusting to the approximate position of a specific warehouse. Second, in order to obtain an accurate transform matrix, we consider using the normal-distributions transform (NDT) (Magnusson et al. 2009) algorithm to accurately match the point cloud data. Similar registration algorithms include the Iterative Closest Point (ICP) (Besl and McKay 1992), but the ICP iterative algorithm needs to provide a better initial value. Simultaneously, due to the algorithm's defects, the final iterative result may fall into a local optimum. NDT is a method of compactly representing the surface of an object. The first step of NDT is to divide the point cloud into cells by k-means clustering (Duda and Hart 1973), calculate the PDF of each cell based on the points in the cell, and assume that this PDF is Gaussian distribution. This PDF can It is understood as the generative process (Magnusson et al. 2009), in another word, of each cell surface point, which is the local model of the measurement points in this cell. For the $\mathrm{N}$-dimensional $\mathbf{x}$ normal random process, the likelihood of having measured $\mathbf{x}$ is

$p(\mathbf{x})=\frac{1}{(2 \pi)^{D / 2} \sqrt{|\Sigma|}} \exp \left(-\frac{(\mathbf{x}-\boldsymbol{\mu})^{\mathrm{T}} \Sigma^{-1}(\mathbf{x}-\boldsymbol{\mu})}{2}\right)$

where $\boldsymbol{\mu}$ and $\Sigma$ denote the mean vector and covariance matrix of the reference scan surface points within the cell where $\mathbf{x}$ lies.

$$
\begin{aligned}
& \boldsymbol{\mu}=\frac{1}{m} \sum_{k=1}^{m} \mathbf{y}_{k} \\
& \Sigma=\frac{1}{m-1} \sum_{k=1}^{m}\left(\mathbf{y}_{k}-\boldsymbol{\mu}\right)\left(\mathbf{y}_{k}-\boldsymbol{\mu}\right)^{\mathrm{T}}
\end{aligned}
$$

where $\mathbf{y}_{k=1, \ldots, m}$ are the positions of the reference scan points contained in the cell.

Using NDT registration, the goal is to find a pose of the current scan, which maximizes the likelihood of the current scan in the reference frame. The optimized parameters are the rotation and translation of the pose estimate of the current scan.Given a set of point of current $X=\left\{\mathbf{x}_{1}, \ldots, \mathbf{x}_{n}\right\}$, a pose $\mathbf{p}$, which is a parameter vector to be optimised, and a transform function $T(\mathbf{p}, \mathbf{x})$ to transform point $\mathbf{x}$ in space by p. The score function for current parameter vector can be formulated as Eq. 3

$s(\mathbf{p})=-\sum_{k=1}^{n} \tilde{p}\left(T\left(\mathbf{p}, \mathbf{x}_{k}\right)\right)$

which the optimal transform parameter vector $\mathbf{p}$ can be iteratively computed.

The 3D scene map of the warehouse is built in advance, and real time LiDAR data needs to be projected to the corresponding warehouse on the scene map. Each time the local point cloud is multiplied by the solved transform matrix to become a point cloud under the global map, expressed as:

$X_{\text {global }}=T\left(\mathbf{p}_{\text {optimal }}, X_{\text {local }}\right)$

where $X_{\text {global }}$ represent the points be projected to 3D map of corresponding warehouse, $\mathbf{p}_{\text {optimal }}$ is optimal transform parameter vector which is computed by NDT, and $X_{\text {local }}$ is point cloud of adding left and right LiDAR calibrated data.

If the point cloud is transformed each frame and then filtered to calculate the volume, it will increase the time for each frame of data to be transformed. Therefore, when calculating the volume, we do not need to transform the data frame every time but make the map rotate to the point cloud's coordinate once. We can transform the map to the point cloud coordinate just once when the program load the map so it is efficiency.

$M_{\text {local }}=T^{-1}\left(\mathbf{p}_{\text {optimal }}, M_{\text {global }}\right)$

$M_{\text {local }}$ represents the map in LiDAR coordinate system, $M_{\text {global }}$ represents the original map in global coordinate system

Calculating the volume of the material needs to remove the spliced LiDAR data wall and ground, and what is left is the volume of the object to be calculated. Because we want to project the LiDAR data to an image, so we construct a filter to remove the wall, ground and outliers.

$\left\{\begin{array}{l}a_{i} * x+b_{i} * y+c_{i} * z+d_{i}=0 \\ y<\text { const } \\ z<\text { const }\end{array}\right.$

where $a_{i}, b_{i}, c_{i}, d_{i}$, are plane parameters and $y, z$ are length and height constraints.

Through these constraints, a filter is formed to remove the LiDAR wall, ground and outliers, and the remaining point cloud data is the sand pile. However, due to the 
impermeability of LiDAR, the back of the sand pile cannot be scanned by LiDAR, resulting in the missing part of the sand pile. If the volume of the sand pile is directly projected, there will be a large error. We project the sand pile data into the mat (it is a data type). Each pixel of the picture represents $0.1 \mathrm{~m} \times 0.1 \mathrm{~m}$. If the area corresponding to the pixel has a height, the average height of belonging is used as the pixel's gray value. If a pixel value is not the corresponding height is that this height is blocked and temporarily filled with 0 . In order to fill up the obscured height, in the study of the angle of repose of the sand pile (Al-Hashemi and AlAmoudi 2018), the model of the sand pile is shown in Fig. 5. Our actual sand pile can also be approximated like this.

The sand pile is approximately symmetrical with respect to the highest point. Traverse each column of the image, if there is a boundary between the height, that is, the non-zero area and the occluded area 0 , then the pixel is the projection pixel of the highest point of the sand pile $\left(x_{i}, y_{i}\right)$, calculate the pixel distance $d$ of the pixel position with the gray value of 0 relative to the highest point of the column, because the sand pile is approximately symmetric, subtract the pixel distance from the column where the highest point pixel is located $d$ can get the symmetrical position $\left(x_{i}, y_{i-d}\right)$ of the highest point of the occluded area about the sand pile, and use the gray value of $\left(x_{i}, y_{i-d}\right)$ as the occluded gray value, That is, as the predicted value of the occluded height, all the pixels of the height 0 of the sand pile area are predicted, and an approximate projection of the entire sand pile can be obtained. Integrate the sand piles behind the grid, expressed by the formula:

$V=\sum_{i=1}^{N} s_{i} \cdot h_{i}$

$s_{i}$ represents the area corresponding to each pixel, and the gray value of the $h_{i}$ pixel is the height of the sand pile. Add up all the pixel values and multiply it by the bottom area represented by each pixel to calculate the volume of the sand pile.

\subsection{The point cloud fusion}

In order to colour the point cloud of the LiDAR, it is necessary to calibrate the extrinsic parameters between the LiDAR and the camera (Dhall et al. 2017; Wang et al. 2017). In this solution, the corners of the calibration board are used as the calibration target. Due to the non-repetitive scanning feature of the Livox LiDAR, the density of the point cloud larger, easier to find the accurate position of the corner point in the LiDAR point cloud (An et al. 2020). The basic principle of calibration is to calculate and obtain the conversion relationship between the $x, y, z$ coordinates of the same target in the LiDAR coordinate system and the $x, y$ coordinates in the camera coordinate system. Because the corner points are obvious targets in the point cloud and photos, this can reduce the calibration error. The calibration steps include calibration of internal camera parameters, calibration preparation and data collection, and calibration of extrinsic parameters. There are many ways to calibrate the internal parameters of the camera (Heikkila and Silven 1997). We use MATLAB tools to calibrate the internal parameters of the camera. The calibration of the camera is a mature tool so this article will not introduce it in detail. Calibration preparation and data collection include: First, the preparation of the calibration scene, using the four corners of the calibration board as the target, choosing a relatively open environment, and ensuring that the LiDAR is more than $3 \mathrm{~m}$ from the calibration board. Second, connect the LiDAR and camera to view the point cloud and record the data packets and photos of the point cloud. Calibration of three extrinsic parameters. In this section, we use a camera and a Livox LiDAR to realize the function of a RGB-D camera. The color of the point cloud is to calculate the corresponding camera pixel coordinates through the $x, y, z$ of the point cloud and the obtained internal and extrinsic parameter matrix, and the RGB information of this pixel is obtained and then assigned to the point cloud for display so that the LiDAR point cloud can display the real color. We project the LiDAR point cloud to the image pixel by pre-known extrinsic calibration value, so as to
Fig. 5 a The sand model. b The real sand pile. According to the model of the sand pile and the actual sand pile, the sand pile can be approximated to be symmetrical, and the shaded area can be predicted

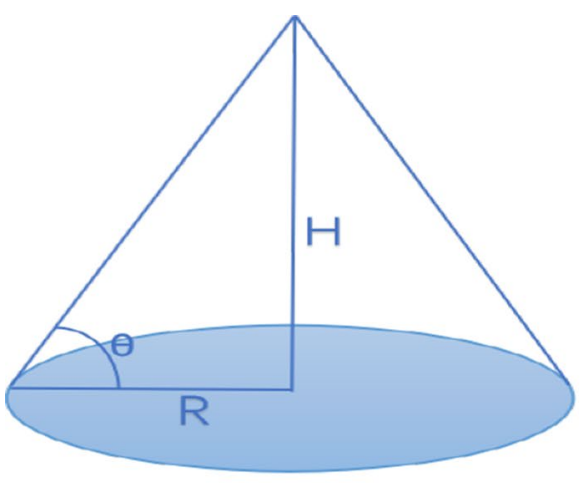

(a)

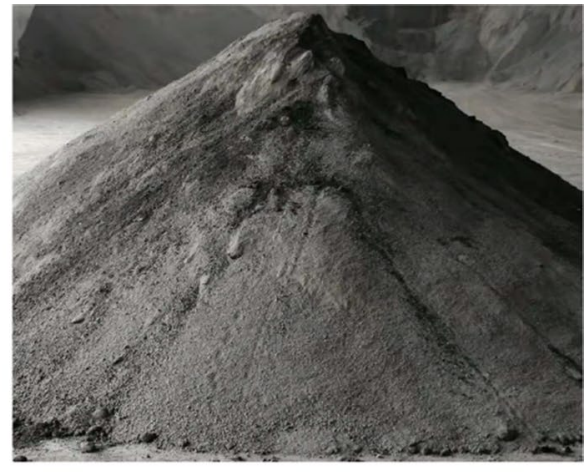

(b) 
colourize the LiDAR point cloud. Then we use a sequence of colourized point clouds to recover a dense colourful map of an area. The advantages of this sensor set are longer detection range and better performance in an outdoor scenario. Data process pipeline as Fig. 6.

\section{Experiment}

The experiment was performed offline by recording rosbag, which is a set of tools for recording from and playing back to ROS topic. We need to calibrate the extrinsic parameters of the two LiDARs and LiDAR point cloud data to the map. The calculated sand pile point cloud is extracted by the fitted plane and height constrain. The filtered point

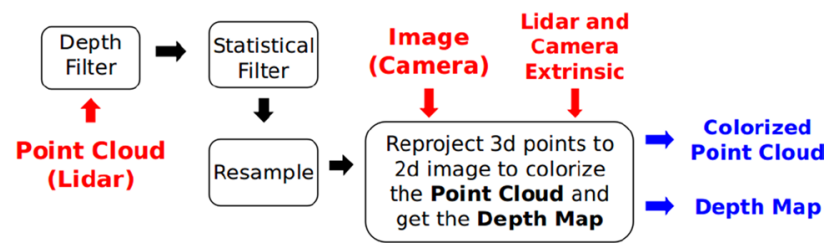

Fig. 6 Data process pipeline of colouring point cloud cloud is projected into the pixels, where each pixel represents a grid of $0.1 \mathrm{~m} \times 0.1 \mathrm{~m}$. After the camera and LiDAR are calibrated, we can use the extrinsic parameters to colour the point cloud. The coloured point cloud has image information and can achieve an effect similar to an RGB-D camera. In this section, the detailed calibration process and results, the prediction results and volume calculation of the occluded area, and the results of point cloud colouring will be introduced.

\subsection{Calibration of extrinsic parameters}

The point cloud collected by two edge computing devices is Fig. 7a. The two LiDARs are based on their respective coordinates as the origin, which causes the data of the two LiDARs to cross and misaligned. It is impossible to calculate accurate extrinsic parameters by directly using the registration algorithm. Because the two edge calculations are fixed on the warehouse and only need to calibrate the extrinsic parameters once, we manually adjust the point cloud on the right to align with the point cloud on the left to get the first extrinsic parameter of the rotation matrix $T_{1}$ Fig. 7b, c, and then use the NDT method to register to get the precise rotation matrix extrinsic parameters $T_{2}$ Fig. $7 \mathrm{~d}$,
Fig. 7 a The raw LiDAR data from distribute edge computing module. b, $\mathbf{c}$ The point cloud manually adjusted to a rough position. d The registered point cloud. Rotating the left point cloud that cannot be registered at all to the right point cloud by manual, two point clouds in a roughly position that can be registered, and then register through the NDT algorithm to obtain a more accurate transform

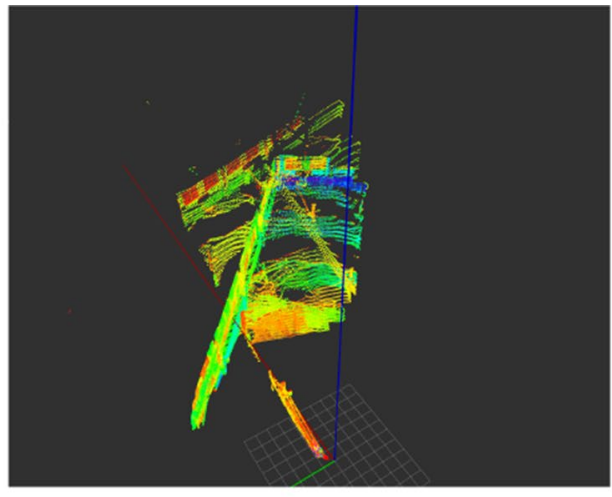

(a)

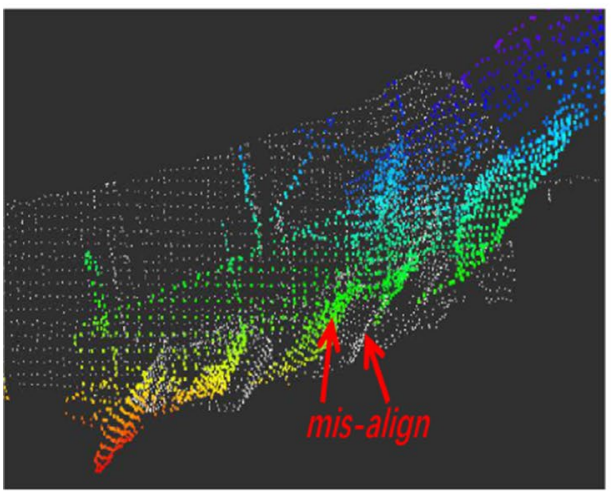

(c)

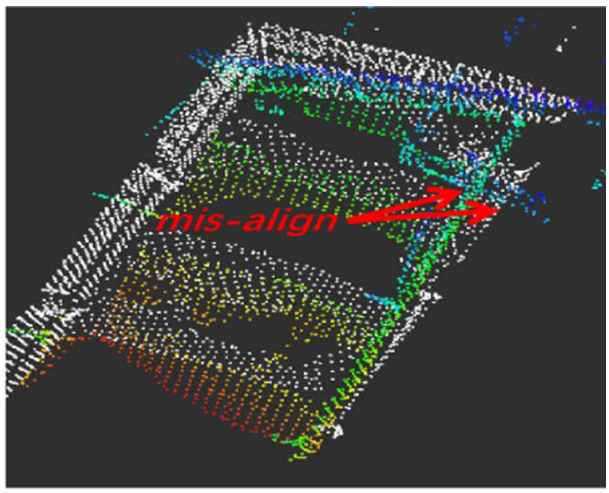

(b)

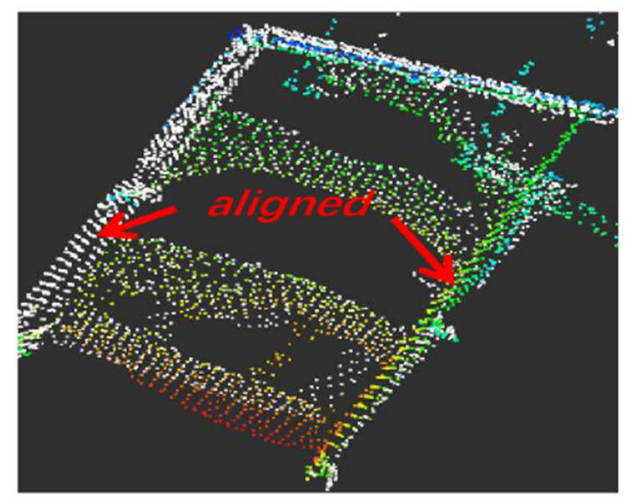

(d) 
so the rotation matrix extrinsic parameters from the right to the left $T=T_{2} \times T_{1}$.

We used GO-ICP (Yang et al. 2015) do experiments without giving the initial value, but LiDAR data can't be matched to the target warehouse. We tried to manually adjust the LiDAR data and map data to a relatively close position but still could not achieve the desired effect. Figure 8 show the result.

Fig. 8 a is manually adjust the LiDAR data and map data. $\mathbf{b}$ is registered through GO-ICP. $\mathbf{c}$ is registered through NDT. Comparing the registration results, using NDT has a better effect than GO-ICP in our environment

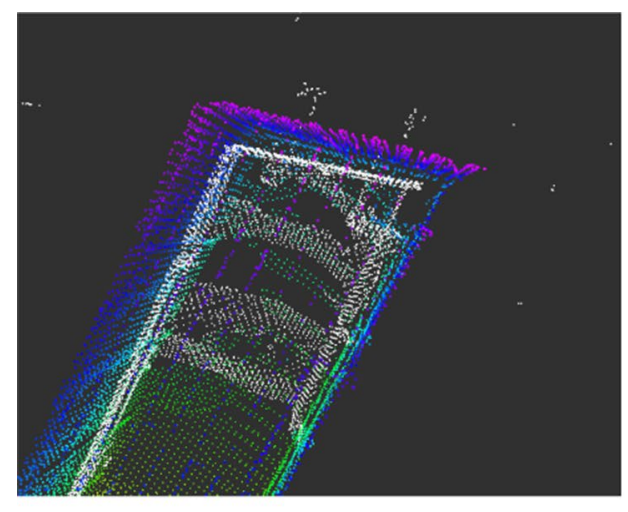

(a)

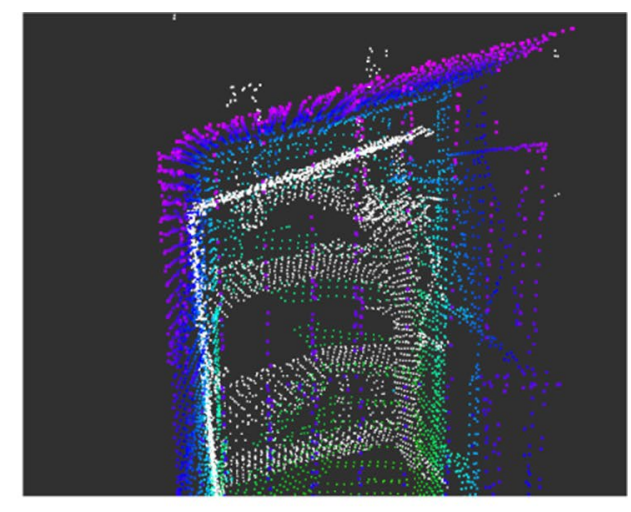

(b)

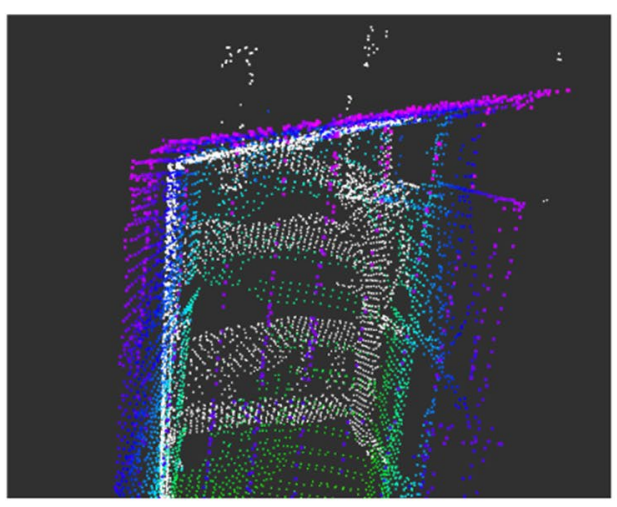

(c)

Fig. 9 a is the sub-map of the warehouse, which is the edge computing module installed. The white point cloud is added point and coloured point cloud is sub-map, both of them were adjusted manually. $\mathbf{b}$ is registered through NDT
In order to facilitate manual adjustment of the point cloud, the calibration is from the LiDAR point cloud data to the map rotation matrix extrinsic parameters $T_{\text {cloud }}^{\text {map }}$. But in the actual material calculation, in order to reduce the amount of calculation and the operation of rotating the point cloud, we only need to rotate the map to the LiDAR point cloud coordinates just once when the program is initialized to load the map. The result of LiDAR point cloud and map calibration as Fig. 9. The operation of rotating 


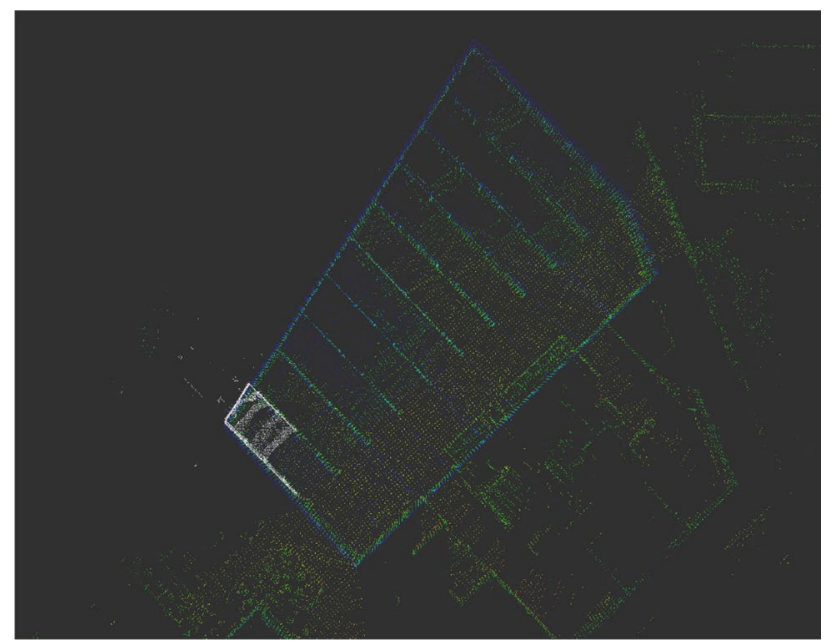

Fig. 10 Top view of rotating warehouse map to edge computing module's LiDAR added point cloud

the map to the LiDAR coordinates is equivalent to multiplying the map by the inverse of the LiDAR to the map $\left(T_{\text {cloud }}^{\text {map }}\right)^{-1}$, formulated as $M_{\text {local }}=\left(T_{\text {cloud }}^{\text {map }}\right)^{-1} \times M_{\text {global }}$, The result be shown as Fig. 10.

\subsection{Point projection and prediction}

The point cloud projection is to project the filtered point cloud into the pixels, and the grey value of each pixel represents the height of a materials. First, we need to determine the range of the point cloud on the $x$-axis and the $y$-axis, and then project the point cloud according to the grid size of $0.1 \mathrm{~m} \times 0.1 \mathrm{~m}$. It is possible that multiple points are projected into one pixel at the same time, so the average value of the height of the multiple points is used as the gray value of the pixel. In actual operation, two pictures of the same size are used. One picture $M_{1}$ stores the total height of all point projections, and one picture $M_{2}$ stores several point clouds projected by each pixel. After traversing all point clouds, $M_{1} / M_{2}$ is the average height corresponding to the grey value of each pixel.

After projecting all point cloud, some pixels may have no point cloud projection in the scan are due to the grid resolution is not small enough. These pixels do not conform to the sand pile model and are not part of the image prediction. The image dilatation is used directly on the image to complete the blank points in the scanned area. The prediction of the point cloud area that cannot be scanned on the back of the sand pile is based on the model that the sand pile is symmetric about the highest point. When traversing the columns of the picture, the edge of each sand pile and the highest point of the sand pile is recorded to complete each sand pile. The pile is symmetric concerning the highest point, so the unscanned area's height can be predicted according to the sand pile model. The distance from the highest point to the area with a gray value of zero is not greater than the distance from the highest point of the sand pile to the edge of the sand pile. After all the columns are traversed, the prediction of the height of the unscanned sand pile is completed Fig. 11.

To evaluate the accuracy of the calculation and the accuracy of the sand pile model prediction, we did two sets of experiments. One group of experiments compares the actual volume changes of the materials and the changes obtained through volume calculations. The other group compares the prediction accuracy of the sand pile models and double times scannable areas. In the actual working environment, each material carried by the forklift is in a range. In our experiment, the actual value of each material is $2.6-2.8 \mathrm{~m}^{3}$. For the accuracy of the calculation, we get the experimental results shown in Fig. 12. The average accuracy reach 0.85 .

For evaluating the accuracy of the sand pile model, we piled up several small sand piles in the material warehouse and scanned them with LiDAR to calculate their volume. Sand pile model achieves high accuracy as Fig. 13.

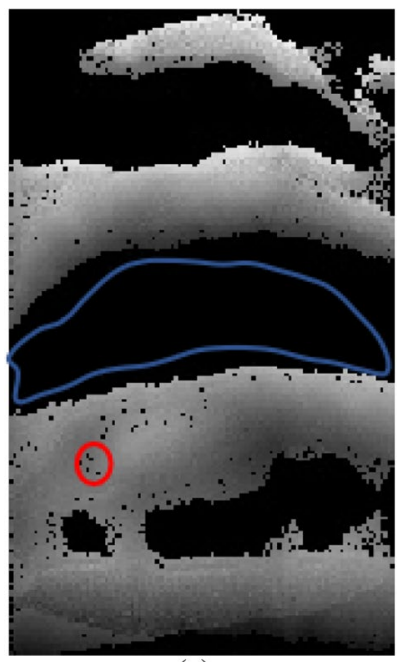

(a)

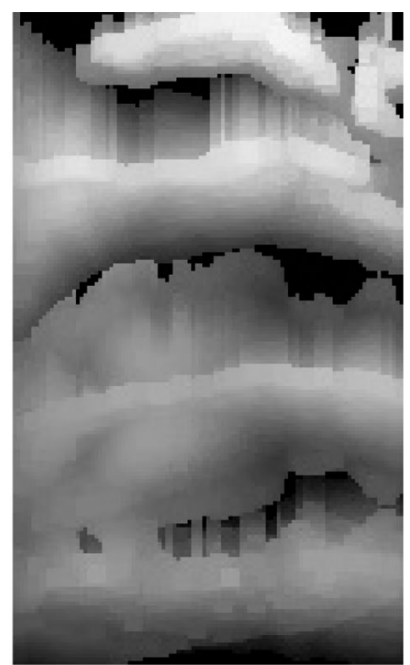

(b)
Fig. 11 a The point cloud be projected to a mat. b The prediction of the sand pile on mat. The red circle area is on the scannable side, so there are no points with gray values because the point cloud is not fully covered during projection. It is not within the range predicted by the sand pile model, and the point height of the scannable area cannot be changed to zero, so the expansion algorithm complements the internal height in the image. The blue area is the area that needs to be predicted based on the model 


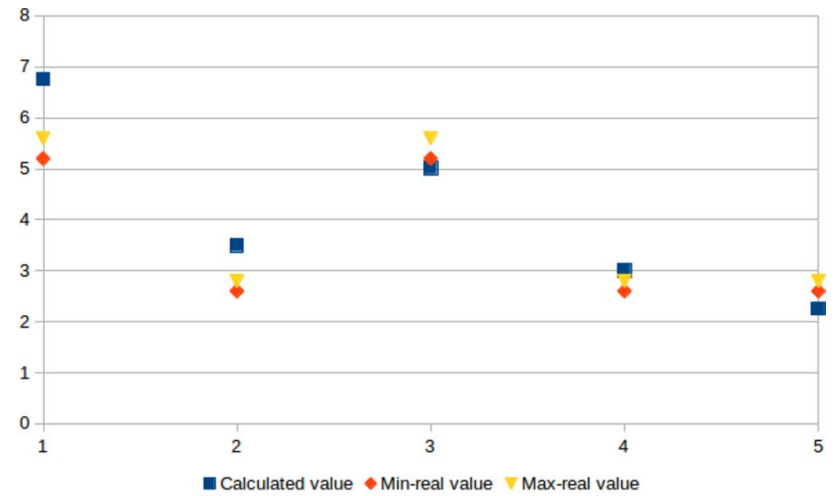

(a)

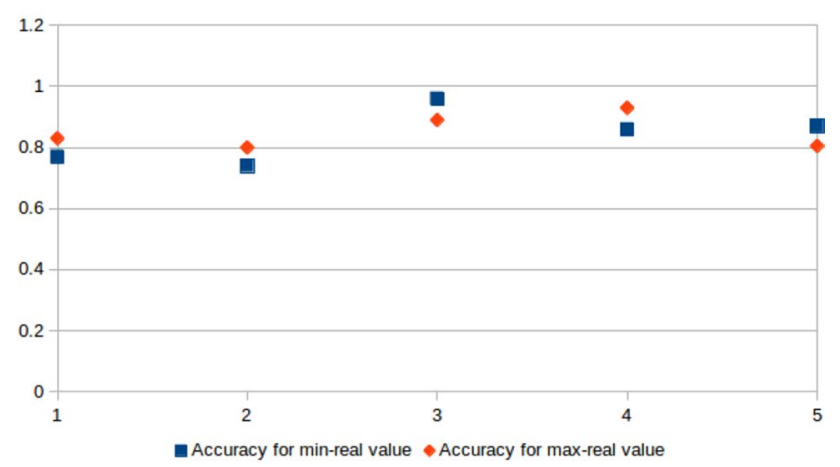

(b)

Fig. 12 a The calculated volume change value and actual volume change value. $\mathbf{b}$ The accuracy of calculation. The material is filled in the warehouse. We remove a part of the material from the warehouse, calculate the changed volume, and calculate the volume calculation method's accuracy

\subsection{LiDAR camera calibration and colouring point cloud}

LiDAR camera calibration is preparation for colouring point cloud. This calibration can be divided into three steps: the first step is the calibration of the camera, the second step is the corner extraction of the LiDAR, and the third step is the calibration of the LiDAR and camera extrinsic parameters. The camera's internal parameters can be easily calibrated for camera calibration using the calibration board and MATLAB tools. The click point selection can be easily selected by clicking the point cloud displayed by the RVIZ (it is a 3-dimensional visualization tool for ROS). Based on this point, the program will extract a plane from the region of interest (ROI) area within a certain range of the point cloud. The corner extraction program will extract corners in this ROI area. The calibration process can be show as Fig. 14. After preparing the camera's internal parameters and the LiDAR's corner points, the extrinsic parameters of LiDAR and camera can be calibrated by the algorithm (Wang et al. 2017). As Fig. 6 show, we input the

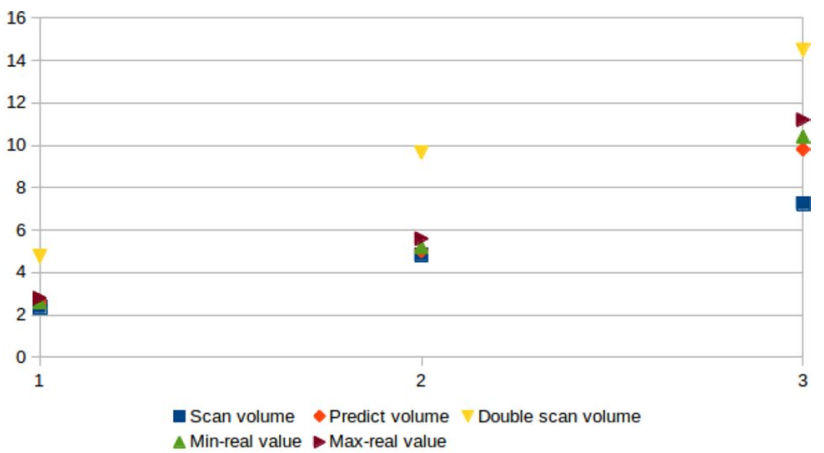

(a)

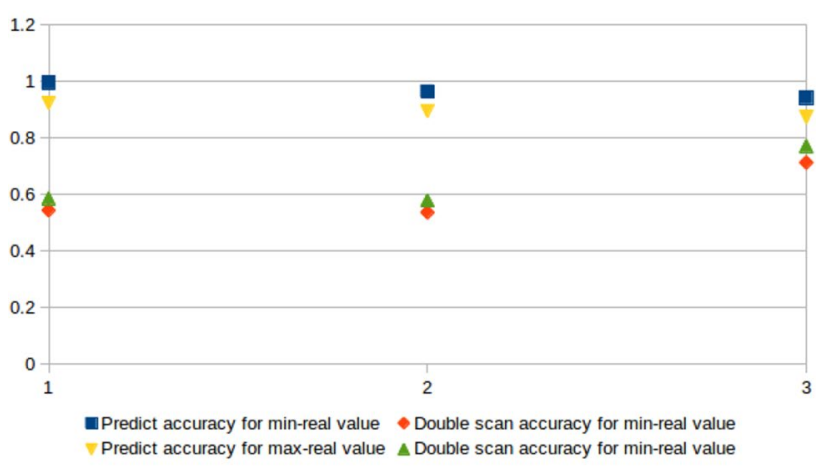

(b)

Fig. 13 a The volume calculated by different methods and the real value. $\mathbf{b}$ The accuracy of the predicted volume based on the sand pile model and the accuracy of twice the scanning volume

camera image and LiDAR camera extrinsic parameters to reproject $3 \mathrm{D}$ points to $2 \mathrm{D}$ image to colourize the point cloud (Fig. 15).

\section{Conclusion}

The intelligent warehouse monitoring system based on edge computing has been initially completed, and the function has been achieved in the calculation of warehouse material volume. We reached the distributed systems and edge computing in unique factories and warehouses that cannot connect directly can form a network through a wireless router. Distributed edge computing can calculate the volume of materials and colour the point cloud. From our experiments, the sandpile model has achieved higher calculation accuracy. It saves the wireless network's bandwidth by transmitting coloured point clouds instead of transmitting pictures and realizes long-distance data transmission. In the future, the primary technology for more comprehensive applications is the network bandwidth and the stability of distributed nodes. What is more, cloud technology is relatively mature, and in the future, 
Fig. 14 a The camera calibration. $\mathbf{b}$ The ROI area depend on the click point. $\mathbf{c}$ The corner extracted by the program. In the c figure, the pink points circled by the green circle are the corner points extracted by the program. Some corner points are not circled

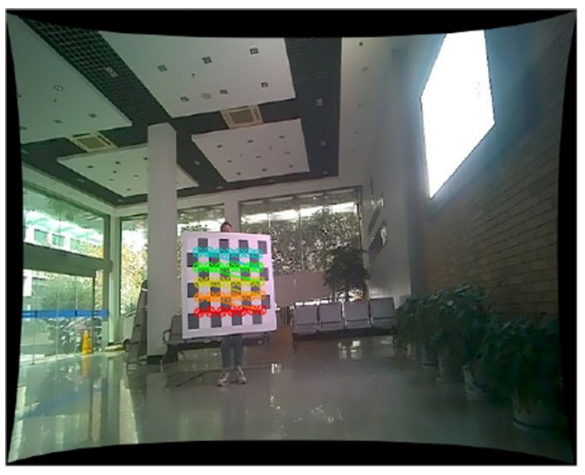

(a)

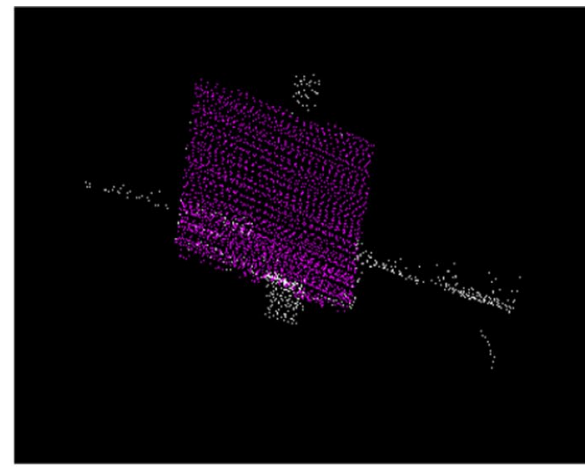

(b)

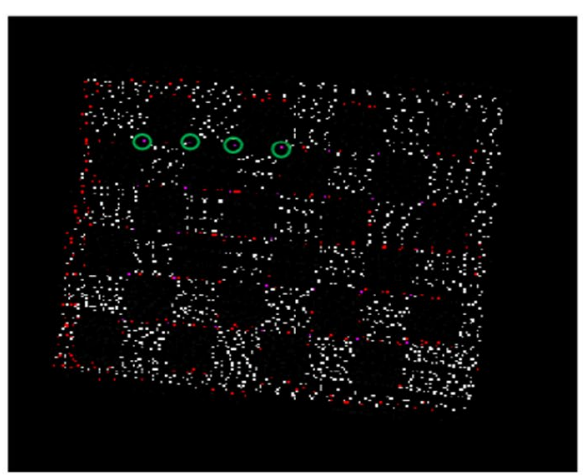

(c)

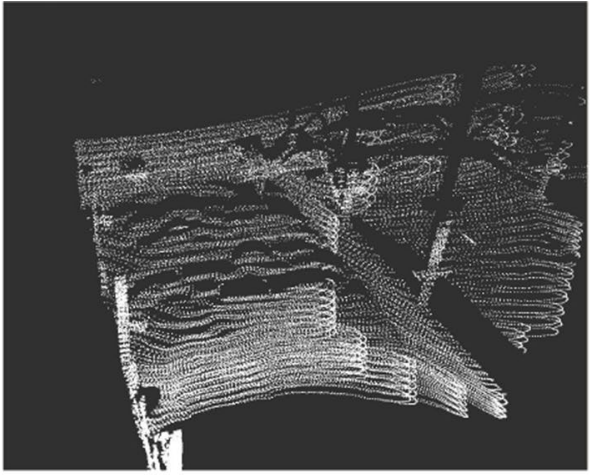

(a)

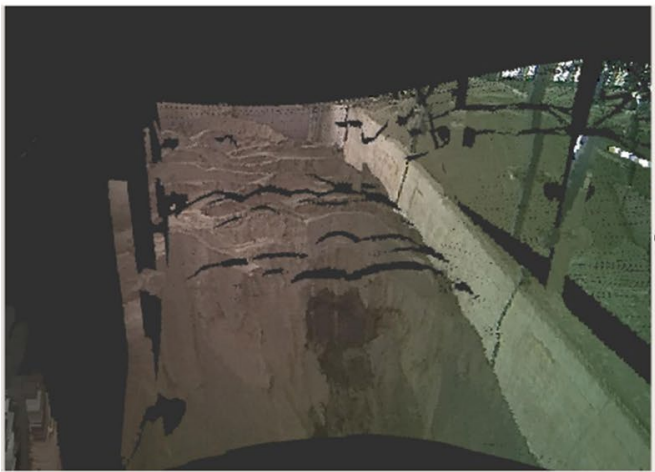

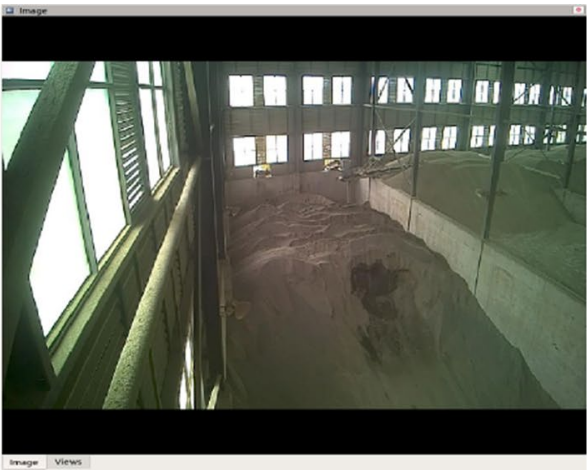

(b)
Fig. 15 a The raw LiDAR data from distribute edge computin canera image point cloud. The point cloud and image can be captured and colourized in edge computing module. The coloured point cloud can be transmitted to the control room for display 
cloud technology can also be used for data transmission across regions and distances.

Acknowledgements Zhejiang Communications Group Inspection Technology Co., Ltd. Hangzhou 310000. Science and Technology Project of Zhejiang Provincial Department of Transport (Number 2020008).

Open Access This article is licensed under a Creative Commons Attribution 4.0 International License, which permits use, sharing, adaptation, distribution and reproduction in any medium or format, as long as you give appropriate credit to the original author(s) and the source, provide a link to the Creative Commons licence, and indicate if changes were made. The images or other third party material in this article are included in the article's Creative Commons licence, unless indicated otherwise in a credit line to the material. If material is not included in the article's Creative Commons licence and your intended use is not permitted by statutory regulation or exceeds the permitted use, you will need to obtain permission directly from the copyright holder. To view a copy of this licence, visit http://creativecommons.org/licenses/by/4.0/.

\section{References}

Al-Hashemi, H.M.B., Al-Amoudi, O.S.B.: A review on the angle of repose of granular materials. Powder Technol. 330, 397-417 (2018). https://doi.org/10.1016/j.powtec.2018.02.003

An, P., Ma, T., Yu, K., Fang, B., Zhang, J., Fu, W., Ma, J.: Geometric calibration for LiDAR-camera system fusing 3D-2D and 3D-3D point correspondences. Opt. Express 28(2), 2122-2141 (2020)

Baheti, R., Gill, H.: Cyber-physical systems. Impact Control Technol. 12(1), 161-166 (2011)

Bauer, Z., Gomez-Donoso, F., Cruz, E., Orts-Escolano, S., Cazorla, M.: UASOL, a large-scale high-resolution outdoor stereo dataset. Sci. Data 6(1), 1-14 (2019)

Besl, P.J., McKay, N.D.: A method for registration of 3-D shapes. IEEE Trans. Pattern Anal. Mach. Intell. 14(2), 239-256 (1992). https:// doi.org/10.1109/34.121791

Chen, B., Wan, J., Shu, L., Li, P., Mukherjee, M., Yin, B.: Smart factory of industry 4.0: key technologies, application case, and challenges. IEEE Access 6, 6505-6519 (2017)

De Brito, M.S., Hoque, S., Steinke, R., Willner, A.: Towards programmable fog nodes in smart factories. In: 2016 IEEE 1st International Workshops on Foundations and Applications of Self* Systems (FAS* W), pp. 236-241. IEEE (2016)

Dhall, A., Chelani, K., Radhakrishnan, V., Krishna, K.M.: LiDARcamera calibration using 3D-3D point correspondences (2017). arXiv preprint. arXiv: 1705.09785

Duda, R.O., Hart, P.E., et al.: Pattern Classification and Scene Analysis, vol. 3. Wiley, New York (1973)

Farkas, L.: High power wireless resonant energy transfer system (2011). US Patent 7,880,337

Fojtík, D.: Measurement of the volume of material on the conveyor belt measuring of the volume of wood chips during transport on the conveyor belt using a laser scanning. In: Proceedings of the 2014 15th international carpathian control conference (ICCC), pp. 121-124. IEEE (2014)

Georgakopoulos, D., Jayaraman, P.P., Fazia, M., Villari, M., Ranjan, R.: Internet of things and edge cloud computing roadmap for manufacturing. IEEE Cloud Comput. 3(4), 66-73 (2016). https:// doi.org/10.1109/MCC.2016.91
Hamilton, E.: What is edge computing: the network edge explained (2018). https://www.cloudwards.net/what-is-edge-computing/. Accessed 14 May 2019

Heikkila, J., Silven, O.: A four-step camera calibration procedure with implicit image correction. In: Proceedings of IEEE computer society conference on computer vision and pattern recognition, pp. 1106-1112. IEEE (1997)

Ikram, W., Thornhill, N.F.: Wireless communication in process automation: a survey of opportunities, requirements, concerns and challenges (2010)

Lee, J., Singh, J., Azamfar, M.: Industrial artificial intelligence (2019). arXiv preprint. arXiv:1908.02150

Liu, X.L., Cao, J.N., Yang, Y.N., Jiang, S.: CPS-based smart warehouse for industry 4.0: a survey of the underlying technologies. Computers 7(1), 13 (2018). https://doi.org/10.3390/computers7010013

Magnusson, M., Andreasson, H., Nuchter, A., Lilienthal, A.J.: Appearance-based loop detection from 3D laser data using the normal distributions transform. In: 2009 IEEE International Conference on Robotics and Automation, pp. 23-28 (2009). https://doi.org/ 10.1109/ROBOT.2009.5152712

Minar, N.: Distributed systems topologies: part 1. In: Emerging Technology Conference (2002)

Newcombe, R.A., Izadi, S., Hilliges, O., Molyneaux, D., Kim, D., Davison, A.J., Kohi, P., Shotton, J., Hodges, S., Fitzgibbon, A.: Kinectfusion: real-time dense surface mapping and tracking. In: 2011 10th IEEE International Symposium on Mixed and Augmented Reality, pp. 127-136. IEEE (2011)

Paavola, M., Leiviska, K.: Wireless sensor networks in industrial automation. In: Factory Automation. IntechOpen (2010)

PDAL Contributors: PDAL point data abstraction library (2018). https://doi.org/10.5281/zenodo.2556738

Pizoń, J., Lipski, J.: Perspectives for fog computing in manufacturing. Appl. Comput. Sci. 12 (2016)

Poonpakdee, P., Koiwanit, J.: Accuracy of distributed systems towards industry 4.0: smart grids and urban drainage systems case studies. Int. J. 14(43), 70-76 (2018)

Riccabona, M., Nelson, T.R., Pretorius, D.H., Davidson, T.E.: Distance and volume measurement using three-dimensional ultrasonography. J. Ultrasound Med. 14(12), 881-886 (1995)

Scheer, A.W.: CIM Computer Integrated Manufacturing: Towards the Factory of the Future. Springer Science \& Business Media (2012)

Shi, W., Cao, J., Zhang, Q., Li, Y., Xu, L.: Edge computing: vision and challenges. IEEE Internet Things J 3(5), 637-646 (2016)

Shu, Z., Wan, J., Zhang, D., Li, D.: Cloud-integrated cyber-physical systems for complex industrial applications. Mob. Netw. Appl. 21(5), 865-878 (2016)

Wang, W., Sakurada, K., Kawaguchi, N.: Reflectance intensity assisted automatic and accurate extrinsic calibration of 3D LiDAR and panoramic camera using a printed chessboard. Remote Sens. (2017). https://doi.org/10.3390/rs9080851

Yang, J., Li, H., Campbell, D., Jia, Y.: Go-ICP: a globally optimal solution to 3D ICP point-set registration. IEEE Trans. Pattern Anal. Mach. Intell. 38(11), 2241-2254 (2015)

Zhang, J., Singh, S.: LOAM: Lidar odometry and mapping in real-time. In: Robotics: Science and Systems, vol. 2 (2014)

Zhongyi, C., Maoji, Y., Bo, K., Xingyuan, B., Junning, C.: Feature parameter extraction algorithm for the large-scale complex structure tank based on $3 \mathrm{~d}$ laser scanning volume measurement. In: 2019 14th IEEE International Conference on Electronic Measurement and Instruments (ICEMI), pp. 651-657. IEEE (2019)

Publisher's Note Springer Nature remains neutral with regard to jurisdictional claims in published maps and institutional affiliations. 

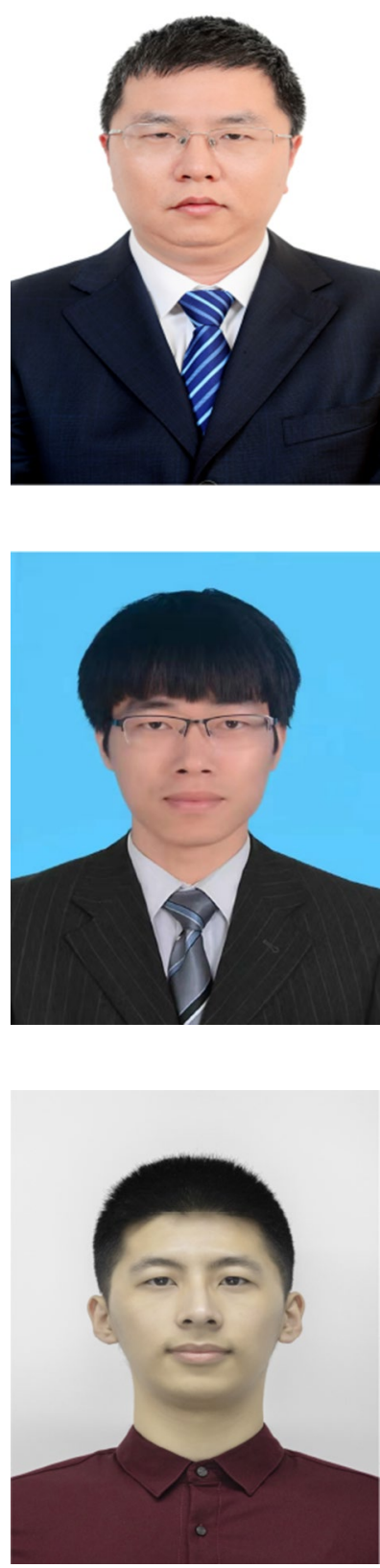

Wenzhou Chen received the B.S. degree in automation from Zhejiang University of Technology in 2017 . He is currently a Ph.D. Candidate of the institute of Cyber Systems and Control, Department of Control Science and Engineering, Zhejiang University. His latest research interests include robot navigation and deep reinforcement learning.

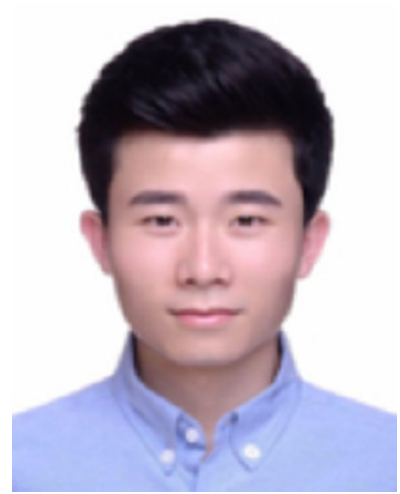

Wenlong Zhou software engineer of Hangzhou BigDataCloudAI Technology Co., Ltd., his recent research interests include robotics and information fusion.

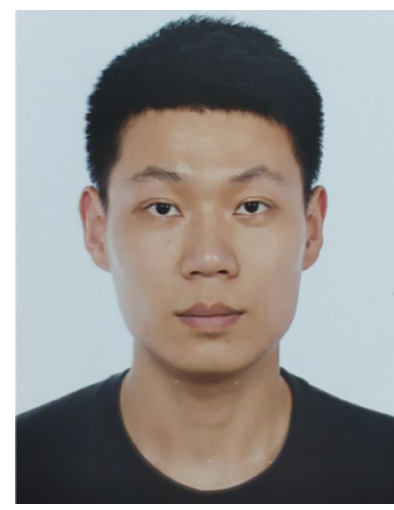

Jinhong $\mathrm{Xu}$ received his MA.Sc degree in Control Science andEngineering from Zhejiang University in 2018. He is currently aresearcher in the institute of Cyber Systems and Control, Departmentof Control Science and Engineering, Zhejiang University. His latestresearch interests include SLAM, information processing and roboticcontrol.

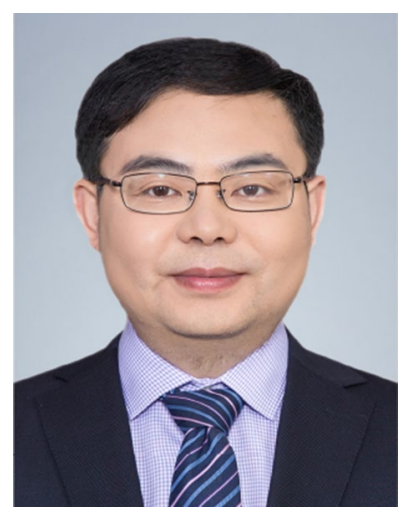

Yong Liu received his B.S. degree in computer science and engineering from Zhejiang University in 2001, and the Ph.D. degree in computer science from Zhejiang University in 2007. He is currently a professor in the institute of Cyber Systems and Control, Department of Control Science and Engineering, Zhejiang University. He has published more than 30 research papers in machine learning, computer vision, information fusion, robotics. His latest research interests include machine learning, robotics vision, information processing and granular computing.

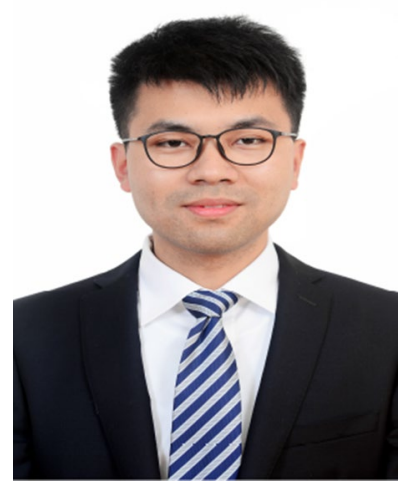

Jinqiang Yao Master's Degree, engineer. Deputy Chief engineer of Zhejiang Communications Group Inspection Technology Co., Ltd., member of China Computer Society. His research directions include Traffic Internet of things, intelligent control, and traffic informatization. He is the corresponding author of this paper. 